\title{
Buprenorphine for Chronic Pain: A Safer Alternative to Traditional Opioids
}

\author{
Suhani Dalal ${ }^{1}$, Ahish Chitneni ${ }^{1}$, Amnon A. Berger, MD, Ph.D. ${ }^{2}$, Vwaire Orhurhu, MD, MPH ${ }^{3}$, Bilal Dar, MD ${ }^{4}$, Bennett \\ Kramer, MD ${ }^{5}$, Anvinh Nguyen, MD ${ }^{6}$, John Pruit, MD ${ }^{6}$, Charles Halsted, MD ${ }^{6}$, Alan D. Kaye, MD, Ph.D. ${ }^{7}$, Jamal Hasoon, \\ $\mathrm{MD}^{8}$ a \\ 1 Department of Anesthesiology, A.T. Still University School of Medicine, ${ }^{2}$ Department of Anesthesia, Critical Care and Pain Medicine, Beth Israel \\ Deaconess Medical Center and Harvard Medical School, ${ }^{3}$ Department of Anesthesiology and Perioperative Medicine, University of Pittsburgh Medical \\ Center, ${ }^{4}$ Department of Anesthesia, Critical Care and Pain Medicine, Brigham and Women's Hospital and Harvard Medical School, ${ }^{5}$ Department of \\ Anesthesia, Critical Care and Pain Medicine, Massachusetts General Hospital and Harvard Medical School, ${ }^{6}$ Department of Anesthesiology, Baylor \\ College of Medicine, ${ }^{7}$ Department of Anesthesia and Pain Management, Louisiana State University Health Sciences Center, ${ }^{8}$ Department of \\ Anesthesia, Critical Care and Pain Medicine, Beth Israel Deaconess Medical Center and Harvard Medical School; Department of Anesthesiology, Baylor \\ College of Medicine \\ Keywords: analgesics, opioids, chronic pain, buprenorphine \\ https://doi.org/10.52965/001c.27241
}

\section{Health Psychology Research}

Vol. 9, Issue 1, 2021

\begin{abstract}
With the ongoing public health crisis with prescription opioids, there is a need for safer alternatives for medication management in chronic pain patients. Buprenorphine is a partial mu-opioid agonist which is commonly utilized to treat patients with opioid-use disorders. The purpose of this review is to discuss the potential use of this medication for the treatment of chronic pain instead of resorting to more traditional Schedule II opioids. Buprenorphine offers a safer alternative for patients who require opioids to manage chronic pain, given the unique pharmacological properties that allow it to provide adequate analgesia with less abuse potential.
\end{abstract}

\section{INTRODUCTION}

Chronic pain continues to affect roughly $30 \%$ of Americans. ${ }^{1}$ The treatment algorithm for most chronic pain conditions typically requires a multimodal approach. Generally, this begins with conservative therapies, including physical therapy, cognitive behavioral therapy, pain psychology, and medication management. Common medications for chronic pain include acetaminophen, non-steroidal anti-inflammatory drugs (NSAIDs), membrane stabilizers, antidepressants, and opioids. Given the ongoing opioid crisis and known risks and side effects of opioids, patients should generally not be started on opioids early in the treatment process for chronic pain conditions. Further supporting this notion is evidence that nearly 11 million people per year in the United States have misused prescription pain relievers, with the most common medications being Schedule II opioids hydrocodone and oxycodone. ${ }^{2}$

However, given the prevalence of chronic pain in the United States, a significant patient population is still prescribed opioids for pain management and quality of life. There are also physicians who continue to prescribe opioids as the mainstay of their practice for chronic pain. ${ }^{3}$ Additionally, a substantial number of patients utilize benzodiazepines in addition to opioid medications, which increases the risk of opioid overdose. ${ }^{4}$ This demonstrates a considerable need for alternative medicine that can provide adequate analgesia while minimizing the risks associated with traditional opioids.

Opioids vary in their efficacy to provide analgesia as well as abuse potential. Most opioids fall into the Schedule II category. Buprenorphine is categorized as a Schedule III medication, indicating that this medication demonstrates less abuse potential than typically used opioids. With the ongoing public health crisis and the fact that a significant number of patients are already on opioid therapy, buprenorphine offers an attractive alternative for patients who require opioid medications for chronic pain.

\section{PHARMACODYNAMICS AND MECHANISM OF ACTION OF BUPRENORPHINE}

Buprenorphine, a lipophilic molecule derivative of the

\footnotetext{
Corresponding author: Jamal Hasoon MD

Address: 1 Brookline PI. Brookline MA 02445

Phone: 617-278-8000

Beth Israel Deaconess Medical Center and Harvard Medical School, Department of Anesthesia, Critical Care and Pain Medicine, Boston, MA
} 
opium alkaloid thebaine, functions as a partial mu-opioid receptor (MOR) agonist and a weak kappa opioid receptor (KOR) antagonist. ${ }^{5}$ In addition, buprenorphine is also known to have agonist activity on the ORL-1 receptor, which aids in the analgesic effect of the drug while lowering adverse effects such as constipation. MOR receptors are critical receptors in the body that were one of the first discovered opioid receptors. Several in vivo studies have also proposed the presence of KOR and ORL-1 receptors in the body. 6 The unique ability of buprenorphine to have partial agonism at the MOR compared to full mu-receptor agonism by drugs such as oxycodone, morphine, and fentanyl allows the medicine to provide analgesia without severe adverse events such as respiratory depression. ${ }^{5}$

The pharmacodynamic properties of buprenorphine are measured by binding affinity and the equilibrium dissociation constant (Ki). The higher binding affinity of the drug results in a low $\mathrm{Ki}$ value. In the case of buprenorphine, the structure and binding position of the drug allows for strong binding affinity compared to other opioids such as hydromorphone, morphine, fentanyl, and oxycodone. In addition to strong binding affinity, buprenorphine functions by having slower dissociation from the binding site, which results in longer-term analgesia as compared to other opioids. ${ }^{5}$

\section{PHARMACOKINETICS OF BUPRENORPHINE}

Buprenorphine has high lipophilicity and potency, which contributes to its overall effectiveness. Transdermal, sublingual, and buccal formulations of buprenorphine are typically utilized as they bypass first-pass metabolism and increase overall bioavailability. In a study conducted by Chin et al., the pharmacokinetic characteristics and bioavailability of sublingual buprenorphine were investigated. In the study, the median Tmax occurred 60 minutes following the administration of sublingual buprenorphine. As for the plasma buprenorphine concentrations, $43 \%$ of participants had quantifiable plasma concentrations within 10 minutes, while $93 \%$ had measurable plasma concentrations 20 minutes after administration. ${ }^{7}$ As far as the half-life properties of sublingual buprenorphine in the study, the terminal halflives ( $\mathrm{T} 1 / 2$ ) for the sublingual buprenorphine was 11.2 hours, the Cmax was $0.74 \mathrm{ng} / \mathrm{mL}$, and the volume of distribution was 170 liters. ${ }^{7}$

Another study conducted by Bai et al. reviewed the pharmacokinetics of single and multiple-dose buccal film formulation of buprenorphine. In the trial, both single-dose and multiple-dose open-label studies were conducted to understand the pharmacokinetic properties of buprenorphine. In the single-dose study, bioavailability was observed to be between $46-51 \%$, with a peak concentration dose occurring 2.5-3 hours after administration. In addition, the mean Cmax of buprenorphine ranged from 0.17 $\mathrm{ng} / \mathrm{mL}$ for the $75-\mathrm{ug}$ dose to $1.43 \mathrm{ng} / \mathrm{mL}$ for the $1200-\mathrm{ug}$ doses. ${ }^{8}$

Ciraulo et al. studied the pharmacokinetics of multiple sublingual buprenorphine trails in dose-escalation trials. In this study, multiple doses of sublingual tablets containing buprenorphine alone or buprenorphine and naloxone were studied. Participants in the study were given escalating doses of buprenorphine from $4 \mathrm{mg}$ to $24 \mathrm{mg}$. Calculations of Cmax, Tmax, and AUCs for both formulations were observed at various doses. In short, the study found that Cmax and AUC increased with dose in the studies except for the $16 \mathrm{mg}$ and $24 \mathrm{mg}$ doses where increases were not significant for the buprenorphine-alone formulation. ${ }^{9}$

These studies demonstrate that the sublingual and buccal route is an efficient delivery system with high bioavailability. Additionally, this medication shows a ceiling effect at higher doses, which is most helpful in mitigating the associated euphoria experienced by traditional opioids and respiratory depression, overdose, and death.

\section{BUPRENORPHINE FOR OPIOID DEPENDENCE AND SUBSTANCE ABUSE}

Per the United States Office of National Drug Control Policy, over 900,000 adults in the United States are opioid-dependent. In the past, methadone has been the treatment of choice for opioid dependence. In the early 2000s, approval was granted for the use of buprenorphine and buprenorphine/naloxone (Suboxone) to treat opioid dependence. ${ }^{10}$ Compared to the traditionally used methadone, buprenorphine has less risk of toxicity with higher doses, has lowered withdrawal symptoms, has less abuse potential, and can be more accessible for office-based treatments as compared to methadone programs which are not widely accessible. Despite several advantages, methadone has been proven to be a lower-cost option and a better option in patients with higher tolerances who may not benefit from buprenorphine. ${ }^{10}$ However, there is significant evidence that demonstrates buprenorphine can be beneficial for the treatment of opioid use disorders as well as withdrawal and craving symptoms associated with methamphetamine use. ${ }^{10,11}$

\section{BUPRENORPHINE IN THE TREATMENT OF CHRONIC PAIN}

Buprenorphine is an opioid with unique pharmacologic properties that make it an advantageous option for treating chronic pain. It has been used as an analgesic and is classified as a Schedule III controlled substance in the United States. ${ }^{12}$ The United States Food and Drug Administration (FDA) has approved a buccal film and transdermal patch for severe pain that requires around-the-clock management with opioids. ${ }^{13,14}$ A meta-analysis conducted in 2018 including 96 randomized control trials and 26,169 participants found that opioids have a statistically significant improvement in pain and physical functioning compared to placebo. ${ }^{15}$ One retrospective study discovered that opioiddependent patients with chronic pain had a 2.3-point reduction of pain on a 0 - to 10 -point numerical rating scale when converted to SL buprenorphine. ${ }^{16}$ Cote et al. conducted a systematic review of the use of buprenorphine for chronic pain. They found numerous potential advantages, including increased efficacy for neuropathic pain, ease of use in elderly patients and those with renal impairment, less immunosuppression compared to other opioids, a ceiling effect on respiratory depression, decreased development of tolerance, and an advantageous safety and side effect profile. ${ }^{17}$ Several other studies have also supported 
the use of buprenorphine for the treatment of chronic pain. ${ }^{18-21}$

In treating chronic pain, physicians typically follow the steps on the World Health Organization (WHO) analgesic ladder, which recommends that analgesics should be given orally with increasing dose and potency until adequate pain control is achieved. ${ }^{22}$ Careful consideration must be taken when prescribing opioids for patients with chronic pain. Guidelines defined by the Center for Disease Control and Prevention (CDC) state that opioids should not be considered a first-line agent in treating chronic pain but should be utilized when the benefits outweigh the risks of treatment. 23

Buprenorphine has been studied alongside other opioid medications such as fentanyl, tramadol, codeine, oxycodone, and morphine to treat chronic pain. ${ }^{21}$ Buprenorphine is often preferred over other opioid analgesics due to its high potency and its ceiling effect on respiratory depression. ${ }^{24}$ Because the risk for respiratory depression does not increase with dosage. Buprenorphine is associated with a lower risk for fatal adverse events when compared to other opioids. ${ }^{24}$ Compared to other opioids, buprenorphine has a lower potential for abuse as it is a partial agonist at the muopioid receptor, providing fewer rewarding effects than full agonists. $^{25}$

Buprenorphine has been studied in its various formulations for the management of chronic pain. Sublingual (SL) buprenorphine is available as a tablet, and recent studies have explored a sublingual wafer. ${ }^{26}$ Lim et al. compared the bioavailability and effectiveness of sublingual buprenorphine wafer to tablet by measuring early quantifiable plasma concentrations at various time points between 10 minutes to 48 hours after administration. They found that the absolute bioavailability of SL buprenorphine wafer was approximately $45.4 \%$, compared to the currently available sublingual tablet (Temgesic), which has a bioavailability of about $35 \% .26$

Buccal buprenorphine (BBUP) provides higher bioavailability than the sublingual formulation. ${ }^{27-30}$ The back layer of the buccal film releases the medication unidirectionally into the buccal mucosa, causing less to be lost in the oral cavity. ${ }^{28}$ BBUP is available in a variety of microgram doses and are administered every twelve hours. ${ }^{31}$ Rauck et al. studied the optimal dose of BBUP in 420 opioid naïve patients with chronic low back pain. ${ }^{32}$ The double-blinded, placebo-controlled trial analyzed pain relief from BBUP in the study population. The study found that $75 \mathrm{mcg}$ twice daily is an appropriate initial dose for opioid naïve patients. Still, nearly half of the study population required $450 \mathrm{mcg}$ twice daily to achieve adequate pain control. ${ }^{32}$ Rauck et al. also studied the efficacy and tolerability of BBUP in opioid naïve patients with moderate to severe chronic low back pain. Patients treated with BBUP were more likely to achieve $\geqslant 30 \%$ pain relief when compared to those receiving placebo. Those treated with BBUP were more likely to report a higher global impression of change and less rescue medication. However, the same study found that there was no statistically significant difference between patients receiving BBUP compared to placebo in the patients achieving $\geqslant 50 \%$ pain relief. 32

The buprenorphine transdermal delivery system (BTDS) delivers buprenorphine through the skin via a patch, allowing for prolonged release of buprenorphine at low doses. ${ }^{33}$ Studies of low-dose transdermal buprenorphine patches have found patches were better tolerated than sublingual buprenorphine and were effective in managing chronic low back pain, osteoarthritis, and other forms of chronic pain. ${ }^{33,34}$ Transdermal buprenorphine is available in $5,7.5$, 10,15 , and $20 \mathrm{mcg} / \mathrm{h}$ doses and is designed to be applied every 7 days. BTDS should be started at the lowest possible dose of $5 \mathrm{mcg} / \mathrm{hr}$ and applied to the upper outer arm, upper chest, upper back, or the side of the chest, and can be gradually increased as needed for pain control. ${ }^{34}$ The BTDS is worn continuously for 7 days, with maximal effect generally achieved after 3 days of continuous application. Following removal of the patch, it is recommended to rotate the application site of the patch, as placing the patch in the same site can lead to increased drug absorption. It is recommended that patches are not applied on the same area for 3-4 weeks. ${ }^{34}$

In its matrix form, BTDS has a lower risk for potential misuse. ${ }^{31}$ Yarlas et al. conducted two randomized, doubleblinded controlled trials to study the effect of BTDS on sleep in patients with chronic low back pain. ${ }^{35}$ The study utilized the Medical Outcomes Study Sleep Scale, which assesses sleep disturbance, sleep adequacy, daytime somnolence, and the Sleep Problems Index (SPI). In their first trial, Yarlas et al. compared 10 and $20 \mathrm{mcg} / \mathrm{h}$ BTDS to placebo, and in the second trial, they compared $20 \mathrm{mcg} / \mathrm{h}$ BTDS to a control of $5 \mathrm{mcg} / \mathrm{h}$ BTDS. Both trials showed small but statistically significant improvement in SPI. ${ }^{35}$ James et al. and Naing et al. found that while sublingual and transdermal buprenorphine produced similar analgesic effects, sublingual buprenorphine was associated with a higher risk of side effects. ${ }^{36,37}$ Adverse effects such as nausea, vomiting, and dizziness were worse in the sublingual formulation of buprenorphine. Conaghan et al. compared BTDS to oral opioids in 966 patients with chronic pain secondary to osteoarthritis. ${ }^{38}$ The study found that patients had improved quality of life with BTDS when compared to oral opioids, cocodamol, and tramadol. ${ }^{38}$

The manufacturer of BTDS, Purdue Pharma, published a study that evaluated 31,533 patients with new prescriptions for BTDS during the study period from 2011 to $2015 .{ }^{39}$ Of this study population, $88 \%$ were dispended opioids in the 6 months before being prescribed BTDS. In the 6 months before initiating BTDS therapy, median opioid use for patients was approximately 50 morphine equivalent doses (MED). Patients used BTDS for a median of 30 days and a mean of 100 days. In the 6 months following the initial prescription of BTDS, $24 \%$ of patients decreased the total units MED. This suggests that BTDS may be beneficial for patients to step down from higher opioid doses. ${ }^{39}$

\section{CLINICAL SAFETY AND EFFICACY}

Numerous studies have been conducted about buprenorphine and its efficacy and safety as an analgesic for patients with chronic pain. A systematic review of ten trials involving 1,190 patients demonstrated that sublingual buprenorphine is effective as an analgesic. ${ }^{17}$ Raffa et al. reviewed 24 studies to better understand the efficacy of buprenorphine 
as an analgesic. Of the 24 studies, 23 showed that buprenorphine is just as effective as morphine, fentanyl, sufentanil, and oxycodone for pain treatment. ${ }^{40}$

Studies have shown that buprenorphine has analgesic properties while also having a ceiling effect on respiratory depression, which can contribute to decreased fatal adverse events compared to other opioids such as fentanyl. ${ }^{41}$ Dahan et al. studied the effects of intravenous buprenorphine and fentanyl on respiratory depression in a randomized, double-blind, placebo-controlled trial. ${ }^{24}$ The study demonstrated that fentanyl produced dose-dependent respiratory depression leading to apnea at doses $\geqslant 3 \mu \mathrm{g} / \mathrm{kg}$, while buprenorphine was shown to have a ceiling effect on respiratory depression at high doses. ${ }^{24}$

Pergolizzi et al. determined that buprenorphine is an essential opioid for use in elderly patients, as its clearance is not affected by age. ${ }^{42}$ Similarly, Al-Tawil et al. decided that there was no difference in clearance of buprenorphine between elderly patients over the age of 70 and younger patients with the average age of 32 who were receiving BTDS $10 \mathrm{mcg} / \mathrm{hr}^{43}$ Buprenorphine has also been studied in patients with renal failure. Filitiz et al. studied the effects of transdermal buprenorphine in patients with severe renal failure and found that plasma clearance of buprenorphine is not altered by renal failure or dialysis. ${ }^{44}$ Use of buprenorphine in patients with liver impairment has also been extensively studied and has shown that mild to moderate liver impairment does not affect the clearance of buprenorphine. ${ }^{45}$ However, mild to moderate liver impairment may impair clearance of naloxone and increase levels; thus, buprenorphine-naloxone combination therapy should be avoided in these patients. ${ }^{46}$ In severe hepatic failure, patients can have increased buprenorphine bioavailability. ${ }^{45}$ Drugs associated with decreased hepatic blood flow may contribute to decreased hepatic clearance of buprenorphine and increased bioavailability. ${ }^{46}$ The pharmacokinetics of buprenorphine metabolism via CYP3A4 isoenzymes may lead to drug interactions with CYP3A4 inhibitors. ${ }^{46,47}$ Protease inhibitors, specifically atazanavir, cause increased bioavailability of buprenorphine, leading to increased CNS depressant effects. Of note, ketoconazole, a CYP3A4 inhibitor, is not associated with increased plasma levels of buprenorphine. ${ }^{46,47}$

Buprenorphine is known to inhibit cardiac repolarization, causing prolongation of the QTc interval. Doses of BTDS $>20 \mathrm{mcg} / \mathrm{hr}$ were noted to cause an increase in the QTc interval, with doses of 40 and $80 \mathrm{mcg} / \mathrm{hr}$ increasing the QTc interval by $12-14 \mathrm{~ms}$. However, buprenorphine has not been associated with arrhythmias or Torsades de pointe. ${ }^{31}$ Buprenorphine should be used with caution with other medications that prolong the QTc interval or those who have a genetically prolonged QT syndrome. ${ }^{48,49}$

The safety and efficacy of buprenorphine has been studied for various etiologies of chronic pain. A double-blind, randomized, placebo-controlled trial by Simpson et al. studied the safety and efficacy of BTDS in 186 patients with diabetic peripheral neuropathy. ${ }^{50}$ The rate of withdrawal from the study due to adverse effects, chiefly nausea and vomiting, was high (39.8\% in the BTDS group and $25.8 \%$ in the placebo group). Of patients who could tolerate BTDS, $86.3 \%$ reported a $\geqslant 30 \%$ reduction in average pain after 12 weeks, compared to $56.6 \%$ in the placebo group. Yoon et al. conducted an open-label study without a placebo to evaluate the safety and efficacy of BTDS in 114 patients with chronic musculoskeletal pain. ${ }^{51}$ Patients were started on 5 $\mathrm{mcg} / \mathrm{hr}$ and titrated up as needed to a maximum dose of $40 \mathrm{mcg} / \mathrm{hr}$ over 6 weeks, and then continued therapy for 11 weeks. The study discovered a statistically significant $(\mathrm{p}<$ 0.0001 ) mean 2.27-point decrease in pain on an 11-point pain scale during the study period. Patients also reported an improvement in sleep quality $(p=0.054)$ and an improvement of overall health state $(p<0.0001)$ after treatment. During the study, $78.1 \%$ of patients experienced treatmentrelated adverse effects. These included nausea (39.5\%), constipation (31.6\%), dizziness (27.2\%), somnolence (19.3\%), vomiting (16.7\%), headache (8.8\%), pruritis $(7.9 \%)$, and application site reactions $(6.1 \%) .{ }^{51}$ Despite some adverse effects in buprenorphine treatment, it has safety advantages that make it useful in the treatment of chronic pain.

\section{CONCLUSION}

Buprenorphine demonstrates unique pharmacological properties that make it an attractive medication for chronic pain patients who require opioid medications. Buprenorphine displays a partial agonist at the mu-opioid receptor, allowing potent analgesia with a better safety profile. Additionally, the medication is a Schedule III opioid with less abuse potential than traditional Schedule II opioids typically used for chronic pain. Practitioners should be aware that this medication is associated with a high proportion of side effects such as nausea, vomiting, and constipation. It is also associated with QTc prolongation, especially at higher doses. However, given the ongoing opioid crisis in the United States, buprenorphine is a valuable tool for treating chronic pain.

Submitted: July 15, 2021 EST, Accepted: July 30, 2021 EST 


\section{REFERENCES}

1. Dahlhamer J, Lucas J, Zelaya, C, et al. Prevalence of chronic pain and high-impact chronic pain among adults-United States, 2016. Morb Mortal Wkly Rep. 2018;67(36):1001-1006. doi:10.15585/mmwr.mm6736 $\underline{\mathrm{a} 2}$

2. Substance Abuse and Mental Health Services Administration. Key Substance Use and Mental Health Indicators in the United States: Results from the 2017 National Survey on Drug Use and Health (HHS Publication No. SMA 18-5068, NSDUH Series H-53).; 2018. https://www.samhsa.gov/data/report/2017-nsd uh-annual-national-report

3. Rosenblum A, Marsch LA, Joseph H, Portenoy RK. Opioids and the treatment of chronic pain: Controversies, current status, and future directions. Exp Clin Psychopharmacol. 2008;16(5):405-416. doi:1 $\underline{0.1037 / \mathrm{a} 0013628}$

4. Sun EC, Dixit A, Humphreys K, Darnall BD, Baker LC, Mackey S. Association between concurrent use of prescription opioids and benzodiazepines and overdose: retrospective analysis. BMJ. 2017;356. doi:1 $\underline{0.1136 / \mathrm{bmj} . j 760}$

5. Gudin J, Fudin J. A Narrative Pharmacological Review of Buprenorphine: A Unique Opioid for the Treatment of Chronic Pain. Pain Ther. 2020;9(1):41-54. doi:10.1007/s40122-019-00143-6

6. Pasternak G, Pan YX. Mu opioid receptors in pain management. Acta Anaesthesiol Taiwan. 2011;49(1):21-25. doi:10.1016/j.aat.2010.12.008

7. Chin Beng Lim S, Schug S, Krishnarajah J. The Pharmacokinetics and Local Tolerability of a Novel Sublingual Formulation of Buprenorphine. Pain Med. 2019;20(1):143-152. doi:10.1093/pm/pnx321

8. Bai SA, Xiang Q, Finn A. Evaluation of the Pharmacokinetics of Single- and Multiple-dose Buprenorphine Buccal Film in Healthy Volunteers. Clin Ther. 2016;38(2):358-369. doi:10.1016/j.clinther a.2015.12.016

9. Ciraulo DA, Hitzemann RJ, Somoza E, et al. Pharmacokinetics and pharmacodynamics of multiple sublingual buprenorphine tablets in dose-escalation trials. J Clin Pharmacol. 2006;46(2):179-192. doi:10.11 $\underline{77 / 0091270005284192}$

10. Donaher PA, Welsh C. Managing opioid addiction with buprenorphine. Am Fam Physician.

2006;73(9):1573-1578.
11. Ahmadi J, Sahraian A, Biuseh M. A randomized clinical trial on the effects of bupropion and buprenorphine on the reduction of methamphetamine craving. Trials. 2019;20(1):1. doi:1 $\underline{0.1186 / \mathrm{s} 13063-019-3554-6}$

12. Webster L, Gruener D, Kirby T, Xiang Q, Tzanis E, Finn A. Evaluation of the tolerability of switching patients on chronic full $\mu$-opioid agonist therapy to buccal buprenorphine. Pain Med (United States). 2016;17(5):899-907.

13. Fishman MA, Kim PS. Buprenorphine for Chronic Pain: a Systemic Review. Curr Pain Headache Rep. 2018;22(12):83. doi:10.1007/s11916-018-0732-2

14. https://www.accessdata.fda.gov/drugsatfda_docs/1 abel/2014/021306s015s019lbl.pdf

15. Busse JW, Wang L, Kamaleldin M, et al. Opioids for Chronic Noncancer Pain: A Systematic Review and Meta-analysis. JAMA. 2018;320(23):2448-2460. doi:1 0.1001/jama.2018.18472

16. Daitch J, Frey ME, Silver D, et al. Conversion of chronic pain patients from full-opioid agonists to sublingual buprenorphine. Pain Phys. 2012;15(suppl 3):ES59-ES66. doi:10.36076/ppj.2012/15/es59

17. Cote J, Montgomery L. Sublingual buprenorphine as an analgesic in chronic pain: a systematic review. Pain Med. 2014;15(7):1171-1178. doi:10.1111/pme.12 $\underline{386}$

18. James IGV, O’Brien CM, McDonald CJ. A randomized, doubleblind, double-dummy comparison of the efcacy and tolerability of low-dose transdermal buprenorphine (BuTrans seven-day patches) with buprenorphine sublingual tablets (Temgesic) in patients with osteoarthritis pain. J Pain Symp Manag. 2010;40(2):266-278. doi:10.1016/j.jpainsymman.201 $\underline{0.01 .013}$

19. Naing C, Yeoh PN, Aung K. A meta-analysis of efficacy and tolerability of buprenorphine for the relief of cancer pain. SpringerPlus. 2014;3(1):87. doi:1 $\underline{0.1186 / 2193-1801-3-87}$

20. Within PJ, Derry S, Moore RA, Stannard C, Aldington D, Cole P, et al. Buprenorphine for neuropathic pain in adults. Cochrane Database Syst Rev. 2015;(9):CD011603.

21. Buprenorphine for Chronic Pain: A Review of the Clinical Effectiveness. Canadian Agency for Drugs and Technologies in Health; 2017. 
22. Ventafridda V, Saita L, Ripamonti C, De Conno F. WHO guidelines for the use of analgesics in cancer pain. Int J Tissue React. 1985;7:93-96.

23. Dowell D, Haegerich TM, Chou R. CDC Guideline for Prescribing Opioids for Chronic Pain - United States, 2016. MMWR Recomm Rep. 2016;65(1):1-49. $\underline{\mathrm{d}}$ oi:10.15585/mmwr.rr6501e1

24. Dahan A. Opioid-induced respiratory effects: new data on buprenorphine. Palliat Med. 2006;20(Suppl 1):s3-s8.

25. Walsh SL, Eissenberg T. The clinical pharmacology of buprenorphine: extrapolating from the laboratory to the clinic. Drug Alcohol Depend. 2003;70(Suppl 2):S13-S27. doi:10.1016/s0376-8716(03)00056-5

26. Lim SCB, Schug S, Krishnarajah J. The Pharmacokinetics and Local Tolerability of a Novel Sublingual Formulation of Buprenorphine. Pain Med. 2019;20(1):143-152. doi:10.1093/pm/pnx321

27. Davis MP. Twelve reasons for considering buprenorphine as a frontline analgesic in the management of pain. J Support Oncol. 2012;10(6):209-219. doi:10.1016/j.suponc.2012.05.00 $\underline{2}$

28. Sullivan JG, Webster L. Novel buccal film formulation of buprenorphine-naloxone for the maintenance treatment of opioid dependence: a 12-week conversion study. Clin Ther. 2015;37(5):1064-1075. doi:10.1016/j.clinthera.2015.0 $\underline{2.027}$

29. Kraan H, Vrieling H, Czerkinsky C, Jiskoot W, Kersten G, Amorij JP. Buccal and sublingual vaccine delivery. J Control Release. 2014;190:580-592. doi:10.1 016/i.jconrel.2014.05.060

30. Mendelson J, Upton RA, Everhart ET, Jacob P III, Jones RT. Bioavailability of sublingual buprenorphine. J Clin Pharmacol. 1997;37(1):31-37. doi:10.1177/00912 $\underline{7009703700106}$

31. Davis MP, Pasternak G, Behm B. Treating Chronic Pain: An Overview of Clinical Studies Centered on the Buprenorphine Option. Drugs. 2018;78(12):1211-1228. doi:10.1007/s40265-018-095 $\underline{3-\mathrm{Z}}$

32. Rauck RL, Potts J, Xiang Q, Tzanis E, Finn A. Efficacy and tolerability of buccal buprenorphine in opioid-naive patients with moderate to severe chronic low back pain. Postgrad Med. 2016;128(1):1-11. doi:10.1080/00325481.2016.112830 7
33. Pergolizzi JV Jr, Taylor R Jr, LeQuang JA, Lara A, Ortiz AH, Iban MAR. Pain Control in Latin America: The Optimized Role of Buprenorphine in the Treatment of Cancer and Noncancer Pain. Pain Ther. 2019;8(2):187-201. doi:10.1007/s40122-019-0126-0

34. Plosker GL. Buprenorphine 5, 10 and $20 \mathrm{LG} / \mathrm{h}$

Transdermal Patch: A Review of Its Use in the Management of Chronic Non-Malignant Pain.

35. Yarlas A, Miller K, Wen W, et al. Buprenorphine transdermal system improves sleep quality and reduces sleep disturbance in patients with moderateto-severe chronic low Back pain: results from two randomized controlled trials. Pain Pract. 2015;16(3):345-358. doi:10.1111/papr.12281

36. James IGV, O’Brien CM, McDonald CJ. A randomized, doubleblind, double-dummy comparison of the efcacy and tolerability of low-dose transdermal buprenorphine (BuTrans seven-day patches) with buprenorphine sublingual tablets (Temgesic) in patients with osteoarthritis pain. J Pain Symp Manag. 2010;40(2):266-278. doi:10.1016/j.jpainsymman.201 $\underline{0.01 .013}$

37. Naing C, Yeoh PN, Aung K. A meta-analysis of efficacy and tolerability of buprenorphine for the relief of cancer pain. SpringerPlus. 2014;3(1):87. doi:1 $\underline{0.1186 / 2193-1801-3-87}$

38. Conaghan PG, O’Brien CM, Wilson M, Schofield JP. Transdermal buprenorphine plus oral paracetamol vs an oral codeine-paracetamol combination for osteoarthritis of hip and/or knee: A randomised trial. Osteoarthritis and Cartilage. 2011;19(8):930-938. doi:1 0.1016/j.joca.2011.03.011

39. Wallace L, Kadakia A. Buprenorphine transdermal system utilization. Postgrad Med. 2017;129(1):81-86. doi:10.1080/00325481.2017.1267537

40. Raffa RB, Haidery M, Huang HM, et al. The clinical analgesic efficacy of buprenorphine. J Clin Pharm Ther. 2014;39(6):577-583. doi:10.1111/jcpt.12196

41. Pergolizzi J, Aloisi AM, Dahan A, et al. Current Knowledge of Buprenorphine and Its Unique Pharmacological Profile. Pain Practice. 2010;10(5):428-450. doi:10.1111/j.1533-2500.2010.00 378.x

42. Pergolizzi J, Böger RH, Budd K, et al. Opioids and the management of chronic severe pain in the elderly: consensus statement of an International Expert Panel with focus on the six clinically most often used World Health Organization Step III opioids (buprenorphine, fentanyl, hydromorphone, methadone, morphine, oxycodone). Pain Pract. 2008;8(4):287-313. doi:10.111 1/j.1533-2500.2008.00204.x 
43. Al-Tawil N, Odar-Cederlöf I, Berggren AC, Johnson HE, Persson J. Pharmacokinetics of transdermal buprenorphine patch in the elderly. Eur J Clin Pharmacol. 2013;69(2):143-149. doi:10.1007/s002 28-012-1320-8

44. Filitz J, Griessinger N, Sittl R, Likar R, Schüttler J, Koppert W. Effects of intermittent hemodialysis on buprenorphine and norbuprenorphine plasma concentrations in chronic pain patients treated with transdermal buprenorphine. Eur J Pain.

2006;10(8):743-748. doi:10.1016/j.ejpain.2005.12.001

45. Nasser AF, Heidbreder C, Liu Y, Fudala PJ. Pharmacokinetics of sublingual buprenorphine and naloxone in subjects with mild to severe hepatic impairment (child-pugh classes A, B, and C), in hepatitis $C$ virus-seropositive subjects, and in healthy volunteers. Clin Pharmacokinet. 2015;54(8):837-849. d oi:10.1007/s40262-015-0238-6

46. Butrans (buprenorphine) Transdermal System for transdermal administration: US prescribing information. http://www.purduepharma.com/pi/presc ription/butranspi.pdf

47. BuTrans 5, 10 and $20 \mathrm{ug} / \mathrm{h}$ transdermal patch: EU summary of product characteristics. http://www.medi cines.org.uk/emc/medicine/16787
48. Baker JR, Best AM, Pade PA, McCance-Katz EF. Effect of buprenorphine and antiretroviral agents on the QT interval in opioid-dependent patients. Ann Pharmacother. 2006;40(3):392-396. doi:10.1345/aph.1 g524

49. Kao DP, Haigney MCP, Mehler PS, Krantz MJ. Arrhythmia associated with buprenorphine and methadone was reported to the Food and Drug Administration. Addiction. 2015;110(9):1468-1475. do i:10.1111/add. 13013

50. Simpson RW, Wlodarczyk JH. Transdermal buprenorphine relieves neuropathic pain: a randomized, double-blind, parallel-group, placebocontrolled trial in diabetic peripheral neuropathic pain. Dia Care. 2016;39(9):1493-1500. doi:10.2337/dc $\underline{16-0123}$

51. Yoon DH, Bin SI, Chan SKC, et al. effectiveness and tolerability of transdermal buprenorphine patches: a multicenter, prospective, open-label study in Asian patients with moderate to severe chronic musculoskeletal pain. BMC Musculoskelet Disord. 2017;18(1):337. doi:10.1186/s12891-017-1664-4 